\title{
Las mariposas como estrategia didáctica en el aprendizaje de la taxonomía básica y reconocimiento de la biodiversidad ${ }^{1}$
}

\author{
Diana Johana Carabalí-Banguero ${ }^{2}$ \\ María Claudia Carabalí ${ }^{3}$
}

Recibido: $21-02-2018$

Aceptado: 21 -10-2018

\section{Resumen}

Las mariposas son un grupo de insectos que genera gran interés en los niños y adolescentes por su colorido y forma, sin embargo, muchos desconocen el papel que cumplen en los agroecosistemas y la importancia de su conservación. En el municipio de Buenos Aires (Cauca), en la etnogranja de la Institución Educativa Agroindustrial Valentín Carabalí se realizaron 8 salidas de campo durante el mes de septiembre de 2016, cada una, con una duración de dos horas. El grupo estuvo conformado por 20 estudiantes del grado 9.1 y 20 estudiantes del grado 9.2. Cada grupo realizó una visita semanal a la etnogranja en la cual, se conformaron subgrupos de 3 estudiantes, que realizaron las colectas utilizando red entomológica. Las mariposas fueron sacrificadas haciendo una leve presión en el tórax y se almacenaron en frascos plásticos. En las aulas, sedesarrollaron clases teóricas sobre la taxonomía y la función ecosistémica de los insectos. También, el montaje y su identificación

\footnotetext{
1. Artículo de investigación científica y tecnológica. Este producto se adscribe dentro de la propuesta pedagógica SABERES MATEMÁTICOS Y ENTOMOLÓGICOS DEL ENTORNO "MEDIADOS POR TIC" EN LA I.E. AGROINDUSTRIAL VALENTÍN CARABALI SEDE PRINCIPAL que adelantan docentes de la Institución Educativa Agroindustrial Valentín Carabalí Sede Principal ubicada en el corregimiento La Balsa, Municipio Buenos Aires (Cauca, Colombia) y es apoyado por la institución educativa.
}

2. Magister en Ciencias-Biología (Universidad del Valle). Bióloga (Universidad del Valle). Docente de la Institución Educativa Agroindustrial Valentín Carabalí Sede Principal, Cauca, Colombia.

Cauca - Colombia.

Correo electrónico: dianajohana16@hotmail.com

3. Especialista en Didáctica de las Matemáticas (Universidad Libre). Licenciada en Matemáticas y Computación (Universidad Antonio Nariño). Docente de la Institución Educativa Agroindustrial Valentín Carabalí Sede Principal, Cauca, Colombia.

Cauca - Colombia.

Correo electrónico: carabalimariaclaudia84@gmail.com 
mediante claves taxonómicas, la cual no fue confirmada por expertos en el tema. Se identificaron 12 especies de mariposas de las familias Nymphalidae y Pieridae, las especies más abundantes en la zona fueron Dryas iulia iulia y Ceratinia tutia. Los estudiantes distinguieron a los insectos de otros artrópodos e identificaron las mariposas más comunes en la zona. Las mariposas son un grupo de insectos que facilita un acercamiento a la taxonomía y permite crear conciencia ambiental sobre la conservación de la fauna en niños y adolescentes.

Palabras clave: insectos, diversidad, educación ambiental.

\section{Butterflies as a didactic strategy in the learning of basic taxonomy and recognition of biodiversity}

\section{Abstract}

Butterflies are a group of insects that generate great interest in children and adolescents for their color and shape, however, many are unaware of the role they play in agroecosystems and the importance of their conservation. In the municipality of Buenos Aires (Cauca), in the ethnogranja of the Valentín Carabalí Agribusiness Educational Institution, eight field trips were made during the month of September 2016, each one, with a duration of two hours. The group consisted of 20 students from grade 9.1 and 20 students from grade 9.2. Each group made a weekly visit to the ethno farm in which subgroups of 3 students were formed, who carried out the collections using an entomological network. The butterflies were sacrificed by placing light pressure on the thorax and stored in plastic jars. In the classrooms, theoretical classes on the taxonomy and the ecosystemic function of insects were developed. Also, the assembly and its identification by taxonomic keys, which was not confirmed by experts in the subject. We identified 12 species of butterflies of the families Nymphalidae and Pieridae, the most abundant species in the area were Dryas iulia and Ceratinia tutia. The students distinguished 
the insects from other arthropods and identified the most common butterflies in the area. Butterflies are a group of insects that facilitate an approach to taxonomy and create environmental awareness about the conservation of fauna in children and adolescents.

Keywords: insects, diversity, environmental education.

\section{Introducción}

Los insectos son el grupo animal que presenta mayor diversidad y abundancia con cerca de un millón de especies descritas (Baranzelli et al. 2014). Los seres humanos tienen distintas concepciones de los insectos de acuerdo a la sociedad o cultura a la que pertenezcan. Estas concepciones están influidas por la abundancia de estas especies, su morfología, los beneficios o perjuicios económicos que generen y el conocimiento de la biología de estas especies (Correa y Delgado 2016). Los insectos dada su abundancia y diversidad, tienen una gran importancia ecológica, económica, social y cultural que es reconocida por los estudiantes (Correa y Delgado 2016).

Las mariposas diurnas pertenecen al orden Lepidoptera, son un grupo de insectos de alta diversidad en el neotrópico (Valencia et al. 2005; RíosMálaver 2007). Tienen un papel ecológico fundamental al transformar la materia orgánica, tienen una taxonomía estable, de fácil observación en campo y son sensibles a los cambios en el medio ambiente por lo cual se utilizan como bioindicadores de la calidad de hábitat (Ríos-Málaver 2007). En la enseñanza los insectos son utilizados como material didáctico (Correa y Delgado 2016).

Este trabajo tuvo como objetivo generar un acercamiento a la taxonomía mediante el reconocimiento de mariposas presentes en la zona, así como fomentar el desarrollo de competencias científicas (de la calle, Malaver, Gallego, Rodríguez, Flórez, Saldaña, 2014) y concientizar sobre la importancia de su preservación. 


\section{Materiales y métodos}

La etnogranja la Valentina, está ubicada en el corregimiento la Balsa (Bue-

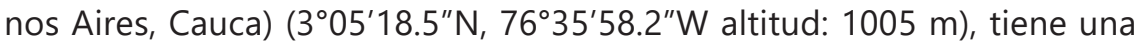
extensión de 3,5 Ha. La temperatura promedio en la zona es de $22^{\circ} \mathrm{C}$ y precipitación media anual es de $2.024 \mathrm{~mm}$; en la zona están sembrados cultivos de plátano Musa paradisiaca L. (Musaceae), yuca Manihot esculenta Crantz (Euphorbiaceae), cebolla Allium cepa L. (Amaryllidaceae) y hortalizas, se presenta un humedal.

Se realizaron 8 salidas de campo durante el mes de septiembre de 2016. El grupo estuvo conformado por 20 estudiantes del grado $9.1 \mathrm{y}$ 20 estudiantes del grado 9.2. Cada grupo realizó una visita semanal a la etnogranja con una duración de dos horas, durante la mañana para capturar las mariposas presentes. Se conformaron grupos de 3 estudiantes, que realizaron las colectas utilizando red entomológica, las mariposas fueron sacrificadas haciendo una leve presión en el tórax. Las muestras rotuladas se almacenaron en frascos plásticos (Figura 1). En las aulas de clases, posteriormente se realizaron clases teóricas sobre la taxonomía de estos grupos y sus funciones en los ecosistemas, el montaje y la identificación de los insectos mediante la clave taxonómica de Valencia et al. (2005).
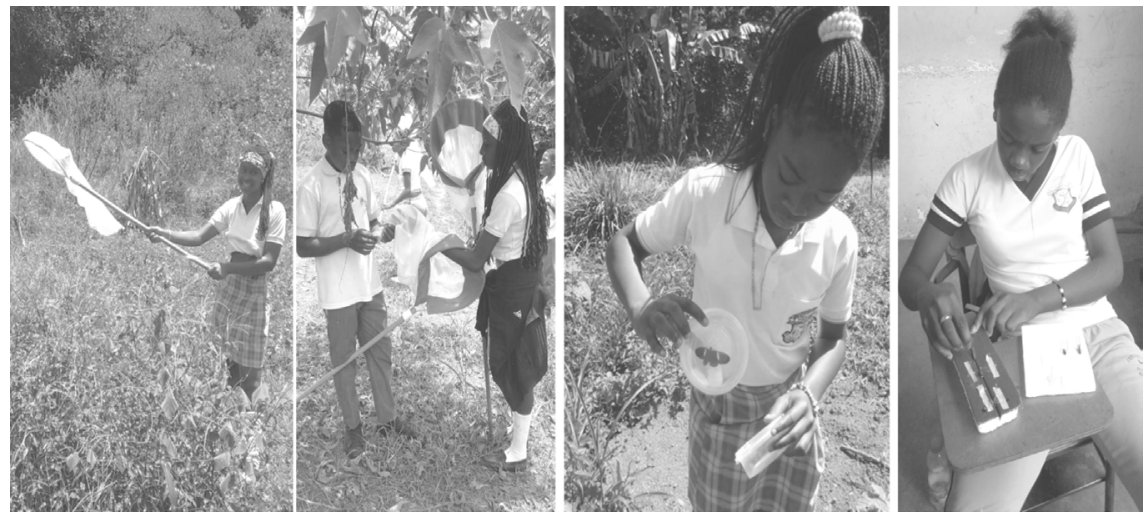

Figura 1. Colecta y montaje de Lepidópteros en la etnogranja la Valentina. 


\section{Resultados}

Se registró un total de 26 mariposas capturadas en la zona de estudio. Se encontraron 12 especies de mariposas reunidos en dos familias: Nymphalidae $(88,46 \%)$ y Pieridae $(11,54 \%)$. El $46,15 \%$ de las mariposas colectadas pertenecen a la subfamilia Heliconiinae $(46,15 \%)$, seguida de Danainae $(23,08 \%)$ Nymphalinae $(19,23 \%)$ y Coliadinae $(11,54 \%)$. Las especies más representativa fueron: Dryas iulia iulia (Fabricius, 1775) y Ceratinia tutia (Hewitson, 1852).

Tabla 1. Listado de especies de mariposas presentes en la etnogranja la Valentina.

\begin{tabular}{|c|c|c|c|}
\hline Familia & Subfamilia & Especie & $\begin{array}{l}\text { Número } \\
\text { de insec- } \\
\text { tos }\end{array}$ \\
\hline \multirow{10}{*}{ Nymphalidae } & Danainae & Ceratinia tutia Hewitson, 1852 & 6 \\
\hline & Heliconiinae & Heliconius erato Linnaeus, 1758 & 1 \\
\hline & Heliconiinae & Actinote equatoria Bates, 1864 & 3 \\
\hline & Heliconiinae & Dryas iulia iulia Fabricius, 1775 & 7 \\
\hline & Heliconiinae & Actinote anteas Doubleday, 1847 & 1 \\
\hline & Nymphalinae & Anartia amathea Linnaeus, 1758 & 1 \\
\hline & Nymphalinae & Anartia jatrophae Linnaeus, 1763 & 1 \\
\hline & Nymphalinae & Siproeta stelenes Linnaeus, 1758 & 1 \\
\hline & Nymphalinae & Hamadryas feronia Linnaeus, 1758 & 1 \\
\hline & Nymphalinae & Adelpha serpa celerio Bates, 1864 & 1 \\
\hline \multirow{2}{*}{ Pieridae } & Coliadinae & Pyrisitia nise venusta Boisduval,1836 & 1 \\
\hline & Coliadinae & Phoebis sennae marcellina Cramer, 1777 & 2 \\
\hline
\end{tabular}

En la etnogranja institucional se desarrolló un trabajo teórico-práctico en el cual, los estudiantes realizaron actividades que favorecieron la adquisición de habilidades científicas. Los estudiantes mostraron motivación y aprendieron a distinguir a los insectos de otros artrópodos. Se generó un aprendizaje significativo (Aparicio, 2018) al motivar la reflexión sobre la importancia ecológica de los organismos presentes en el ecosistema que habitan (Figura 2). 

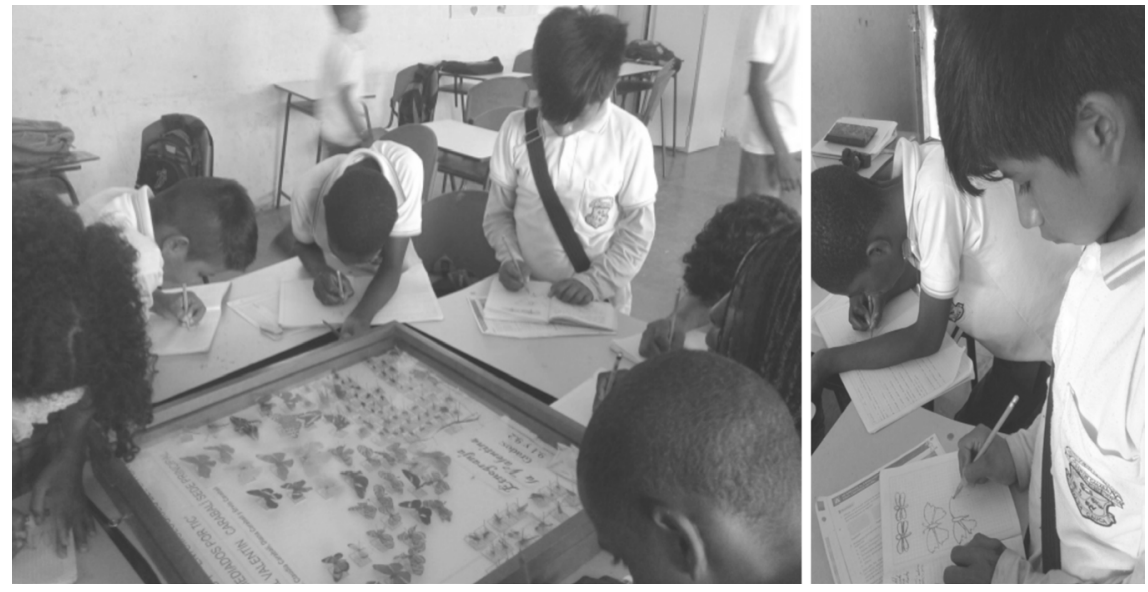

Figura 2. Identificación de lepidópteros colectados en la Etnogranja la Valentina.

La práctica de campo permitió que llevaran a cabo las etapas del proceso científico, principalmente la observación y el planteamiento de interrogantes (Langer, 2016). El tamaño, patrón de coloración, forma de las alas, amplitud alar les permitió el reconocimiento de estos organismos, además de conocer las condiciones ambientales y de hábitat que podrían favorecer su presencia. Se concientizaron a los estudiantes sobre la importancia de la identificación y preservación estos organismos.

\section{Discusión}

Es posible que la abundancia de la familia Nymphalidae se deba a la presencia de plantas nutricias de inmaduros y adultos. Dentro de esta familia, las subfamilias: Danainae, Heliconiinae y Nymphalinae, muestran preferencia por áreas abiertas, con disponibilidad de néctar floral, frutos en descomposición, sales minerales disueltas y excrementos (Ríos-Málaver 2007). La especie Dryas iulia iulia (Heliconiinae) fue la más abundante posiblemente por la presencia de plantas arvenses en la etnogranja, las cuales se ha reportado que frecuenta esta especie (Valencia et al. 2005). La subfamilia Danainae en el neotrópico tiene especies que presentan mimetismo mulleriano con especies de Ithomidae (García-Robledo et al. 2002).

Los adultos de la familia Pieridae se reconocen por su coloración clara, generalmente blanca, amarillenta o naranja, estos insectos están 
presentes en una gran variedad de hábitats consumiendo néctar. La subfamilia Coliadinae presenta distribución neotropical, particularmente la especie, Phoebis sennae es común en áreas abiertas con altitud menor a los 1700 m.s.n.m. alimentándose de sales minerales y néctar floral (Valencia et al. 2005; García-Robledo et al. 2002).

Las mariposas por sus características morfológicas facilitan el aprendizaje de la taxonomía. Los adultos de la familia Nymphalidae tienen el primer par de patas atrofiado lo cual facilita distinguir estos insectos de otras familias. A la vez, desde el punto de vista ecológico: los colores aposemáticos, la presencia de manchas ocelares y patrones de coloración de algunas especies permiten la enseñanza en campo del concepto de mimetismo como el batesiano, el anillo mulleriano y la coloración disruptiva que son estrategias de defensa para evitar la depredación (Valencia et al. 2005). No obstante, se presenta dificultad en la incorporación de actividades de identificación de insectos en las aulas debido a la alta riqueza de especies que presentan muchos sitios de muestreo, las similitudes morfológicas entre especies distintas, es decir especies crípticas, o las variaciones dentro de una misma especie (Scheuch et al. 2018).

Dado que los estudiantes presentan una valoración positiva de las mariposas respecto a otros grupos de insectos (Correa y Delgado 2016). Se puede concientizar sobre la importancia de conservar sus hábitats sembrando cercas vivas con sus plantas hospederas, permitiendo la presencia de arvenses e implementando prácticas agrícolas sostenibles en las cuales se realicen bajas aplicaciones de agroquímicos y de espectro reducido y un adecuado manejo de arvenses (Valencia et al. 2005).

\section{Conclusión}

El reconocimiento de la diversidad de mariposas es una estrategia didáctica que permite el desarrollo de competencias en el área de las ciencias naturales. Se logró concientizar sobre la importancia ecológica de preservar la biodiversidad de este grupo de organismos; para lo cual, el reconocimiento de su morfología fue fundamental (Aparicio \& Ostos, 2018). Este es el primer trabajo que registra la diversidad de mariposas de la zona realizando su identificación estudiantes de secundaria. 


\section{Agradecimientos}

Los autores agradecen a María Mery Peña y Jaime Restrepo directivos docentes de la I.E. Agroindustrial Valentín Carabalí; a los docentes de la institución, especialmente a Ervin Andrés Carabalí y Diocelina Mina por su apoyo logístico, y a los estudiantes del grado $11^{\circ}$ promoción 2018.

\section{Referencias bibliográficas}

Aparicio, O.Y. (2018). La investigación escolar. Revista Interamericana de Investigación, Educación y Pedagogía, RIIEP, 11(2). https://orcid.org/0000-0003-3535-6288

Aparicio, O.Y., \& Ostos, O.L. (2018). El constructivismo y el construccionismo. Revista Interamericana de Investigación, Educación y Pedagogía, RIIEP, 11(2). https://orcid. org/0000-0003-3535-6288. https://orcid.org/0000-0002-6477-9872

Baranzelli, M. C., Córdoba, S., Cocucci, A., Glinos, E., y Paiaro, V. (2014). Dime cómo comes y te diré quién eres: una experiencia didáctica para conocer los aparatos bucales de los insectos. Revista de educación en biología, 17(2): 76-85.

Correa, M.R. y Delgado, C. (2016). Los insectos desde la percepción de los estudiantes "Tikuna" y mestizos de educación básica del Municipio de Caballo Cocha. Ciencia Amazónica (lquitos) 6(2): 109-115.

de la Calle, C. V., Malaver, M. O., Gallego, J. D. M., Rodríguez, M., Flórez, J. C., Henao, C. E. \& Saldaña, R. (2014). Aportes de los doctorados de educación en ciencia, tecnología y sociedad, desde la sistematización de sus investigaciones doctorales científicas y formativas, 2000-2010. Revista Interamericana de Investigación, Educación y Pedagogía, RIIEP, 7(1). DOI: https://doi.org/10.15332/s1657-107X.2014.0001.04

García-Robledo, C., Constantino, L.M., Dolores, M. y Kattan, G. (2002). Mariposas comunes de la cordillera Central de Colombia. Programa de Colombia de Wildlife Conservation Society. Cali: Feriva, S.A. 130 p.

Langer, E. (2016). La construcción de confianza para el estudio de prácticas de resistencia en la escolarización de jóvenes en contextos de pobreza urbana. Revista Interamericana de Investigación, Educación y Pedagogía, RIIEP, 9(2). DOI: https://doi. org/10.22490/25391887.1945 
Ríos-Málaver, C. (2007). Riqueza de especies de mariposas (Hesperioidea \& Papilionoidea) de la quebrada "El Águila" cordillera central (Manizales, Colombia). Boletín científico museo de historia natural 11: 272-291.

Scheuch, M., Panhuber, T., Winter, S., Kelemen-Finan, J., Bardy-Durchhalter, M. y Kapelari, S. (2018). Butterflies \& wild bees: biology teachers' PCK development through citizen science. Journal of Biological Education, 52(1): 79-88.

Valencia, C. A., Gil, Z. N., y Constantino, L. M. (2005). Mariposas diurnas de la zona central cafetera colombiana: Guía de campo. 241 p. 